which there have been injected a large variety of ideas and much practical understending by the activities of the Society. This is not to disregard the contributions made by that other large but silent body of expert opinion containing the practitioners of the technologies involved, who for upwards of a generation have provided a technical basis upon which smokeless combustion practice may be achieved. Without the 'know how' to burn fuel smokelessly, legislative action would be ineffective. The need at the present time is the problem of effective application of this existing knowledge.

The conference this year has provided interesting descriptions of recent progress in domestic heating by centralized methods, in particular of the Westminster City Council's district-heating scheme, considered from the financial, engineering and operational aspects. The scheme shows a saving of coal as well as an overall economy, a reduction of atmospheric pollution and improvement in domestic amenities.

Floor warming was an ancient practice known to communities as wide apart as the early Koreans and the Romans. To-day, with the application of electrical heating, the principle of thermal storage may be applied, the electricity being supplied to concrete flooring in 'off-peak' periods. Gradually, but surely, the hardy and reluctant dwellers of the British Isles are succumbing to the lure of the use of partial central heating, an interesting new feature of this practice being the application of forced circulation of hot water in small pipes - a result of research by the British Coal Utilization Research Association. All the heat from the boiler is kept within the living space of the house and is localized mainly in the radiators as the piping is so small. Another interesting article explains how the stuffiness of ducted hot air, another practice emanating from the Romans, may be safeguarded by suitable control of the movement of the air. The above are merely a selection of the many interesting points emerging from the technical discussions of the conference.

On the issues involved in the projected Clean Air Bill, opinions were many and comprehensive, ranging from contributions by the representatives of local authorities to several informative explanations of the purport of the Beaver Report by members of the Committee. These make much more intelligible reading than does the draft of the Clean Air Bill itself. Proverbially, the draft of a Parliamentary Bill in any case provides a form of mental persecution for the layman. The contributions to the Bournemouth conference give at least a clear indication of the intentions of the Report, and the reactions of much representative opinion from the local author. ities. The views of the ordinary man and of the industrialist were unfortunately not heard; this is to be regretted, since they may be regarded in some respects as the victims for the sacrifice.

The objectives of the conference were, as would be expected, altruistic and worthy. The factors of practicability of some of the intentions may be quite another matter, about which, it is to be hoped, something may at last be heard in the debates in Parlia. ment, if the victims have not become mesmerized. by the certainty of a kill. Nevertheless, it must be agreed that now there looms before us some prospect of wise action which may contribute materially to a cleaner Britain that is so much desired by all. How much cause for thanksgiving may emerge in next year's conference.

R. J. SARJANT

\section{CAMBRIDGE ASTRONOMICAL SYMPOSIUM}

THE symposium on "Astronomical Seeing", held at the Observatories, University of Cambridge, during September 12-14, was timed to follow the General Assembly of the International Astronomical Union at Dublin, and thus to take advantage of the presence in Europe at this time of astronomers from all over the world. Its object was to provide a forum for the exchange of ideas and information relating to the various aspects of the subject. These aspects include the accumulated knowledge of visual observers; the extensive ad hoc instrumental studies that have been made more recently, especially in the United States; the theory of scintillation, developed mainly with particular reference to radio problems ; and meteorological information about inhomogeneity and turbulence in the atmosphere. The aim was to provide time in the programme for sufficient discussion, so that progress could be made towards the resolution of real differences.

Dr. J. S. Hall (U.S. Naval Observatory, Washington, D.C.) described experiments in which correlations had been found between intensity scintillation in certain frequency-ranges and the speed of the upper winds. Lamps attached to balloons showed less scintillation even at a height of $30,000 \mathrm{ft}$. than did stars observed at the same time ; but it is probably unsafe, he said, to conclude from the experiments so far made that appreciable seeing effects come from greater heights. Dr. Hall showed a film of shadow bands crossing the objective of the Washington 40-in. telescope, and of extra-focal Hartmann patterns. Dr. P. B. Fellgett (Cambridge Observatories) reviewed the physical theory of scintillation. Plane wavefronts of starlight are diffracted by irregularities in the refractive index of the air, and the diffracted beams interfere to give phase- or amplitude-scintillation according to their mutual phases, which depend on the distance from the irregularities. Brightness-scintillation is usually negligible just below the irregularities, and increases with distance, while phase scintillation decreases slightly. The complex autocorrelation function of the amplitude across the mean direction of travel remains constant; and the mean intensity distribution in a telescopic image is also independent of distance from the source of disturbance. The spatial frequencies in the scintillation differ in general from those in the irregularities of refractive index. Dr. A. Hewish (Cavendish Laboratory) presented quantitative results for some chosen cases. $\mathrm{He}$ showed calculations and slides of the detailed development of brightness changes beyond sinusoidal phase-changing layers of various amplitudes; of the interchange between brightness changes and phase-scintillation; and of the corre. sponding autocorrelation functions of complex amplitude, showing the dominance of harmonics of the layer-frequency when the phase change is large. The corresponding statistical developments were shown for a layer having a Gaussian autocorrelation function of refractive index.

Dr. G. Keller (Perkins Observatory, Ohio) gave a detailed account of work at the Perkins Observatory. Observations have been made of the temporal powerspectra of brightness changes for various telescope apertures; the correlation of scintillation in certain frequency-ranges with upper-wind speed; correlation of upper-wind direction with the dependence of 
scintillation, observed through a slit covering the objective, on the position-angle of the slit; and the degree of coherence between scintillation in selected frequency-ranges observed in two neighbouring apertures. Dr. W. H. Steavenson (Cambridge) discussed the effects which can be distinguished visually in extrafocal images or when the eye is placed at the focus of the telescope. The 'high seeing' appears as shadow patterns blown at high speed across the aperture, and defocusing experiments indicate an average height of origin of about $10 \mathrm{~km}$. Slower shadow bands originating within a few hundred feet of the telescope can be seen to move with the surface wind. Local dome and tube effects are recognizable by their geometry. In large reflectors, the cooling of the mirror causes disturbances in the thin layer of air at the face of the mirror ; these are much more serious than the well-known changes in mirror asphericity and can often be the dominant cause of image spread. Dr. A. Hynek (Ohio State University, Columbus) reported studies of image motion made by Dr. A. G. Wilson at Flagstaff, Arizona. Star-trails recorded on fast-moving film showed the amount of movement, the proportion of the time that the image is sharp, and the occurrence of multiple images. These studies were made with special reference to the design of methods of photographing Mars, and an important conclusion is that at Flagstaff the instantaneous image movement is nearly the same over the whole disk of the planet. Dr. Hynek also reported some very recent work at Ohio State University which included measures of image movement in two colours. If image-spread were an effect of rapid image motion, this might provide a further test of the remark of Hewish and Keller that the red images may be expected sometimes to have a sharper 'core' than the blue.

Dr. B. H. Briggs (Cavendish Laboratory), describing radio observations of the scintillation at neighbouring wave-lengths, said that in the ray-interference approximation the correlation across the spectrum is simply related to the spread in the ray path-lengths between the source and observer. Rapid frequencychange experiments yield records similar to the spectrum-scintillation photographs of Zwicky, and the problems of interpretation are also similar. Dr. E. H. Linfoot (Cambridge Observatories) took up the question of measuring the harm done by bad seeing. Seeing effects reduce the rate at which a given telescope can record information about the sky. The loss can be estimated in terms of the mean transmission factors (sine-wave response factors), which are essentially equal to the mean autocorrelation function of the complex displacement across the aperture. Dr. Linfoot showed how the reduction in information capacity (in Shannon's sense) can be calculated from the statistics of seeing; generally speaking, the loss is of second order when the phaseerrors are small. Dr. R. Goody (Imperial College of Science and Technology, London) presented meteorological data on turbulence and convection in the atmosphere. By day, in clear weather, convection is most intense near the ground and falls rapidly throughout the first $1,000 \mathrm{ft}$. Convection is enhanced over large mountains and islands. A small island should have good daytime seeing, and even a few feet of height above the ground could be beneficial. Aircraft results show inhomogeneities at various heights. A few accelerometer records from balloon flights show increased turbulence near the tropopause, but it is not known whether this is typical. The northern edge of the jet stream in the northern hemisphere is sometimes very turbulent. Generally, however, the atmosphere is non-adiabatic and is continually being mixed, so that the causes of seeing effects are unlikely to be confined to any narrow range of height.

In the discussion, widely different opinions were expressed about the usefulness of the ray approximation to the Huyghens wave treatment. One view is that ray theory is adequate under suitable conditions for small phase perturbations. It was urged in reply that ray theory is essentially the limit of wave theory as the wave-length becomes very small compared with the relevant geometrical pathdifferences, so that it is expected to be valid (as in aberration calculations) only for large phase-changes. It was the general opinion that the phase-changes corresponding to good seeing could be of the order of a few radians, but the lack of any specific measurements of this fundamental quantity illustrates how much is still to be learnt. It was pointed out that the combined effect of spatial smoothing by the objective aperture, and temporal smoothing in the electrical filters, would in general make the amount of scintillation recorded photoelectrically dependent on the transverse speed of the shadow bands. Unitil this is allowed for, it cannot be said whether the observed wind-scintillation correlation represents an actual physical connexion between wind-speed and optical inhomogeneities in the air. Non-isotropic inhomogeneities would necessarily give rise to telescopic images that are not radially symmetrical, although this effect might be masked by disturbances originating at another height. It was suggested that the height of origin of scintillation could be found in principle from the cross-over wave-length in the colour scintillation; from the ratio of phase to brightness-scintillation at different wave-lengths; and by the analogue of the spaced-receiver experiments of Briggs et al., the linear transverse velocity being found from the correlation of scintillation in two apertures and the angular velocity by observa. tions of two neighbouring stars. A plea was made for observations in narrower wave-length ranges.

\section{HUNGARIAN-CZECHOSLOVAK POLAROGRAPHIC CONFERENCE}

TOR the first Hungarian-Czechoslovak PolaroIraphic Conference held in Veszprém, Hungary, during August 11-14, seventeen Czechoslovak polarographists, mostly from the Polarographic Institute of the Czechoslovak Academy of Science, were guests of the modern Chemical University in Veszprém at the meeting arranged jointly by the University, the Hungarian Chemical Society and the Hungarian Academy of Science. Prof. J. Heyrovský was the leader of the Czechoslovak delegation; from the Hungarian side, Dr. Polinszky (dean), Dr. Preissich (secretary of the Chemical Society), Dr. Cieleszky (chairman of its Polarographic Section), and Prof. Bognár (general secretary of the Hungarian Academy) organized the Conference extremely successfully. The Minister of Education, Prof. Erdey-Grúz, a prominent physico-chemist, was in the chair. At the opening of the Congress, a diploma of honorary fellowship of the Hungarian Academy of Science was presented to Prof. J. Heyrovský, who then delivered an address on the "New Trends in Polarography". Prof. L. 